\title{
Adaptive Fuzzy Ho Robust Control For Manipulators In MIMO Systems
}

\author{
Xinming $\mathrm{Hou}^{\mathrm{a}}{ }^{\star}$, Yanhua Lei ${ }^{\mathrm{b}}$, Hongtao $\mathrm{Yu}^{\mathrm{c}}$ and $\mathrm{Yan} \mathrm{Bao}^{\mathrm{d}}$
}

College of Automation, Shenyang Institute of Engineering, Shenyang Liaoning110136, China

ahou_xinming@sina.com, blyhw127@163.com, neu970773@sohu.com, ddianzixinxi2004@163.com

Keywords: Manipulators; Robust Control; Uncertain Items

Abstract. An adaptive fuzzy sliding mode robust control algorithm based on fuzzy compensation is proposed for the uncertain items, such as friction, unknown disturbances. An adaptive fuzzy system is used to approximate the unknown nonlinear term, the parameter adaptive law of fuzzy system is designed by using Lyapunov function, eliminating the dependence on prior knowledge. Finally, the effectiveness of the control algorithm is verified by the simulation experiment of the two degree of freedom manipulator.

\section{Introduction}

Robot is a highly complicated system with time-varying, strong coupling and nonlinear dynamic characteristics. It also contains various uncertain factors, such as parameter measurement errors, load changes, external disturbances and the friction between joints, etc. These uncertainties will affect the control performance and dynamic quality of the system seriously. The control studies for uncertain robot with modeling error and external disturbance are particularly important.

\section{System Description}

Consider a manipulator governed by the following dynamic model:

$$
\ddot{x}=F(X)+G(X) u+d
$$

Where $X=\left[\begin{array}{ll}x^{T} & \dot{x}^{T}\end{array}\right]^{T} \in \mathfrak{R}^{n}$ is the state vector, observable; $x \in \mathfrak{R}^{n}$ is the output vector; $F(X) \in \mathfrak{R}^{n}, G(X) \in \mathfrak{R}^{n}$ are nonlinear functions. $d$ contain the coupling term and the disturbance;

Assumption1: $d$ is bounded signal, the upper bound is $\bar{d}$, and $d \in L_{2}[0,+\infty)$.

Assumption2: Suppose that $G(X)$ is a nonsingular matrix, that is, $G^{-1}(X)$ exists.

Define the tracking error of system as:

$$
e=x-x_{d}
$$

The m-th fuzzy is : $R^{(m)}$ : if $x_{1}$ is $A_{1}^{m}$, 且 $x_{n}$ is $A_{n}^{m}$, then $y$ is $B^{m}(\mathrm{~m}=1, \ldots \mathrm{N})$.

Where $A_{i}^{m} \in\left\{A_{i}^{1}, \ldots, A_{i}^{m}\right\}, i=1, \ldots, n$ and $B^{m}$ are the input and output linguistic variables of fuzzy set, defined by $x_{i}, \quad y \cdot N=\prod_{i=1}^{n} n_{i}$ is the amount of fuzzy rules.

If we utilize the singleton fuzzifier, product fuzzy inference and central-average defuzzifier, then

$$
y=\hat{f}(x, \theta)=\zeta^{T}(x) \theta
$$

Where $\theta=\left[\begin{array}{llll}\theta^{1} & \theta^{2} & \ldots & \theta^{N}\end{array}\right]^{T}$ is parameter vector, $\zeta(x)=\left[\begin{array}{llll}\zeta_{1}(x) & \ldots & \zeta_{N}(x)\end{array}\right]^{T}$ is regression vector. $\zeta_{k}(x)=\frac{\prod_{i=1}^{n} \mu_{A_{i}^{k}}(x)}{\sum_{k=1}^{N}\left(\prod_{i=1}^{n} \mu_{A_{i}^{k}}(x)\right)}, k=1, \ldots, N \cdot \mu_{A_{i}^{k}}(x)$ is the membership function. 
Lemma1: Fuzzy logic system (4) is a universal approximator, for any continuous function defined on $D \subset \mathfrak{R}^{n}$, and any given constant $\varepsilon_{f}>0$, there exists a fuzzy control system described by Eq.4 satisfying $\sup _{x \in D}|f(x)-\hat{f}(x, \theta)|<\varepsilon_{f}$.

The fuzzy system to approximate the nonlinear function $f_{i}(x), g_{i j}(x)$ is

$$
\hat{f}_{i}\left(x, \theta_{f i}\right)=\left(\xi_{f_{i}}(x)\right)^{T} \theta_{f i}, i=1,2, \quad \hat{g}_{i j}\left(x, \theta_{g i j}\right)=\left(\xi_{g_{i j}}(x)\right)^{T} \theta_{g i j}, i=1,2
$$

Where $\xi_{f_{i}}$ and $\xi_{g_{i j}}$ are fuzzy basis function vectors, $\theta_{f i}$ and $\theta_{g i j}$ are adaptive parameter vectors. $\theta_{f i}{ }^{*}$ and $\theta_{g i j}{ }^{*}$ are the optimal approximation parameter of $\theta_{f i}$ and $\theta_{g i j}$ correspondingly, $\tilde{\theta}_{f i}$ and $\tilde{\theta}_{g i j}$ are approximation errors, $\varepsilon_{f_{i}}(x)$ and $\varepsilon_{g_{i j}}(x)$ are minimum fuzzy approximation error.

$$
\begin{aligned}
\theta_{f i}{ }^{*}=\arg \min _{\theta_{f i}}\left\{\sup _{x \in D_{x}}\left|f_{i}(x)-\hat{f}_{i}\left(x, \theta_{f i}\right)\right|\right\}, \theta_{g i j}{ }^{*}=\arg \min _{\theta_{g i j}}\left\{\sup _{x \in D_{x}}\left|g_{i j}(x)-\hat{g}_{i j}\left(x, \theta_{g i j}\right)\right|\right\}, \tilde{\theta}_{f i}=\theta_{f i}{ }^{*}-\theta_{f i}, \\
\tilde{\theta}_{g i j}=\theta_{g i j}{ }^{*}-\theta_{g i j}, \varepsilon_{f_{i}}(x)=f_{i}(x)-\hat{f}_{i}\left(x, \theta_{f i}\right), \varepsilon_{g_{i j}}(x)=g_{i j}(x)-\hat{g}_{i j}\left(x, \theta_{g i j}\right) \\
u_{0}=[\hat{G}(x)]^{-1}\left[\ddot{x}_{d}-k_{2} e-k_{1} e-\hat{F}(x)\right]
\end{aligned}
$$

$k_{1} k_{2}$ satisfy polynomial $h(S)=S^{2}+k_{2} S+k_{1}$, all the roots in the left half plane of the $S$ plane.

The adaptive control law is

$$
\dot{\theta}_{f_{i}}=-\eta_{f_{i}} \xi_{f_{i}} s_{i}, \dot{\theta}_{g_{i j}}=-\eta_{s_{i j}} \xi_{g_{i j}} s_{i} u_{0 j}
$$

Where $s=\Lambda E, E=\left[\begin{array}{ll}e^{T} & \dot{e}^{T}\end{array}\right]^{T} \in R^{m n}$, adaptive gain $\eta_{f_{i}}$ and $\eta_{g i j}$ are positive constant.

Considering the coupling term and the disturbance, the control law is:

$$
u=u_{0}+u_{h}
$$

Let $E=\left[e^{T}, \dot{e}^{T}\right]$, then Eq.6 is obtained:

$$
\dot{E}=A E+B u_{h}-B \omega^{\prime}
$$

Where, $A=\left[\begin{array}{cc}0 & 1 \\ -k_{1} & -k_{2}\end{array}\right], B=\left[\begin{array}{l}0 \\ 1\end{array}\right]$.Assume that there exists matrix $P=P^{T}>0, Q=Q^{T}>0$ satisfying $A^{T} P+P A^{T}+Q+P B\left(\gamma^{-2}-2 R^{-1}\right) B^{T} P=0$.

Where $\gamma>0$ is the given disturbance rejection index, $R=R^{T}>0$ is the control gain, satisfying $2 \gamma^{2} \geq R$. Then the Robust controller can be designed as

$$
u_{h}=-R^{-1} B^{T} P E
$$

\section{Control Design}

Theorem1: For the multi-input and multi-output nonlinear system described by Eq.2, satisfying the assumption 1-4, design the composite controller Eq.10, among them linear feedback control Eq.7, the approximation of the nonlinear function Eq.5, Eq.6, adaptive parameter adjustment law Eq.8, Eq.9 and $H \infty$ robust compensation control law Eq.13, then the closed-loop system is stable, and all signals of the closed loop system are bounded.

Proof: select the following Lyapunov function: 


$$
V=\frac{1}{2} s^{T} s+\frac{1}{2} \frac{1}{\eta_{f}}\left[\tilde{\theta}_{f}^{k}\right] \tilde{\theta}_{f}^{k}+\frac{1}{2} \frac{1}{\eta_{g}}\left[\tilde{\theta}_{g}\right] \tilde{\theta}_{g}
$$

According to adaptive control law Eq.8, Eq.9,

$$
\dot{V}=-s\left[\varepsilon_{f}(x)+\varepsilon_{g}(x) u_{0}+G(x) u_{h}+d\right] \leq 0
$$

Thus, the closed-loop system is stable.

\section{Illustrative Examples}

The control law is used in the system equation in [5].The goal of system control is to enable the actual output of the system to accurately track the desired motion trajectory, and the robot's joint position and velocity tracking error can converge to zero asymptotically. Choose the desired trajectory $x_{d 1}=\sin t+\sin 2 t, x_{d 2}=0.5 \sin t+\cos 2 t, x_{1}(0)=x_{2}(0)=0.1 \mathrm{rad}, \dot{x}_{1}(0)=\dot{x}_{2}(0)=0 \mathrm{rad} / \mathrm{s}$, $w=1.5+2 e+5 \dot{e}$.

Fig. 1 and Fig. 2 are position tracking performance of the two joints under the control action of adaptive fuzzy robust control algorithm. Fig.3 and Fig.4 show the speed tracking performance of the two joints. Fig.5 and Fig. 6 are the control input signals of the two joints respectively.
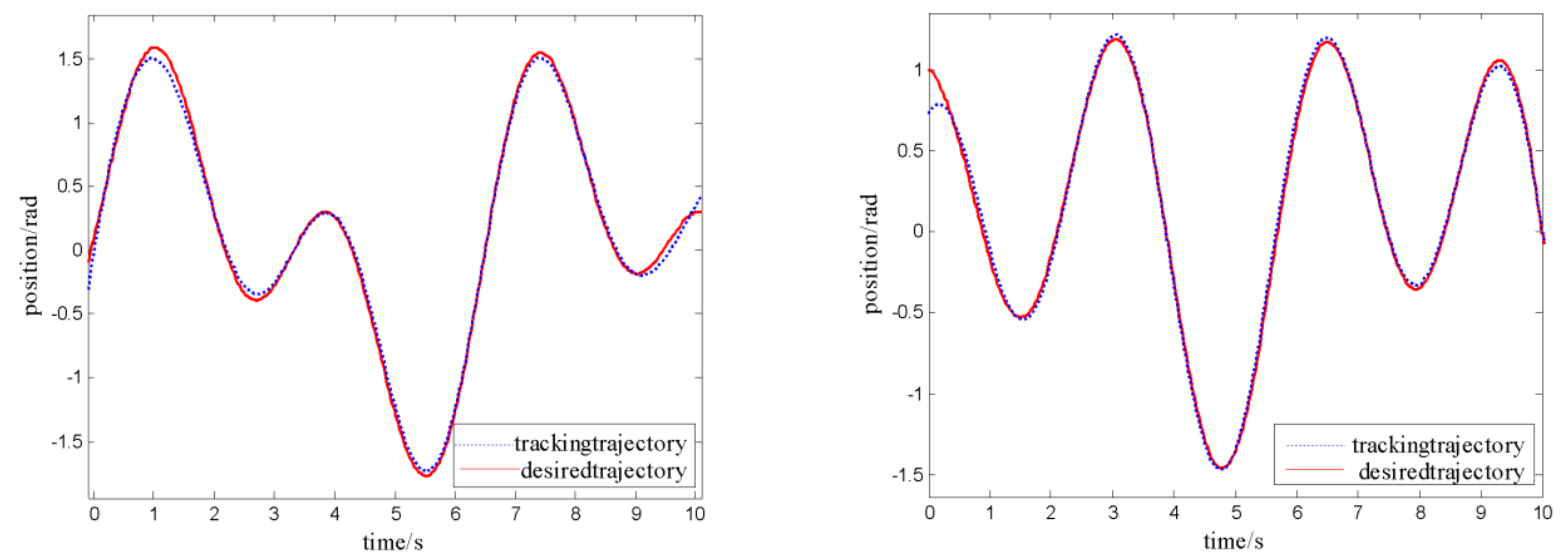

Figure. 1 position tracking performance of joint 1 Figure. 2 position tracking performance of joint 2

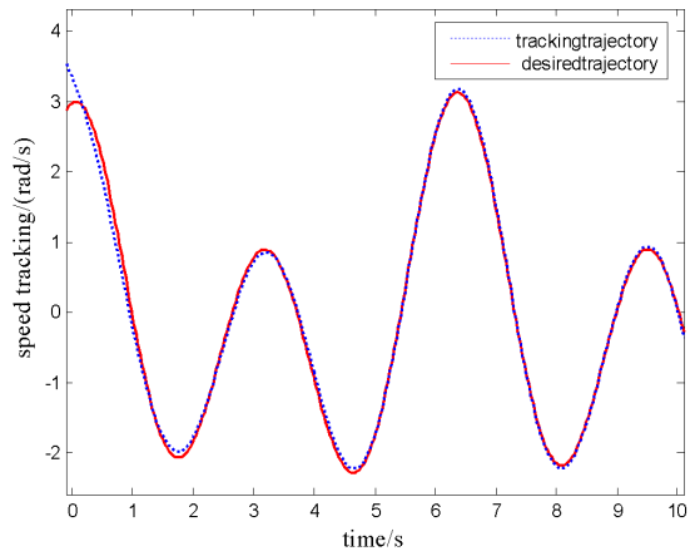

Figure. 3 speed tracking performance of joint 1

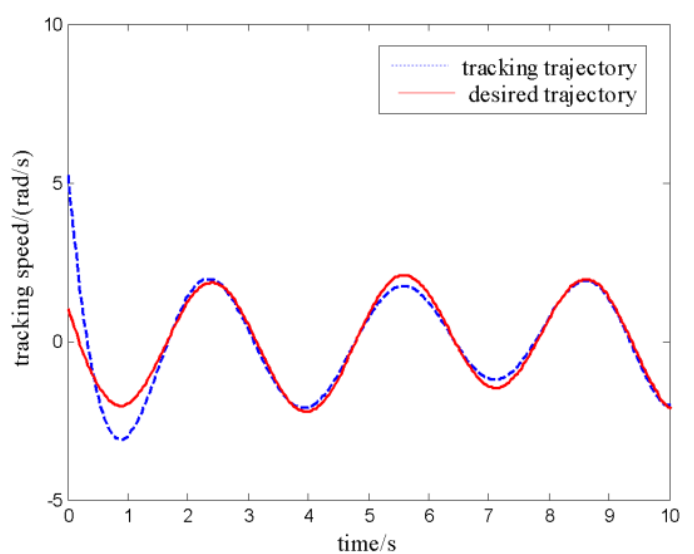

Figure.4 speed tracking performance of joint 2 


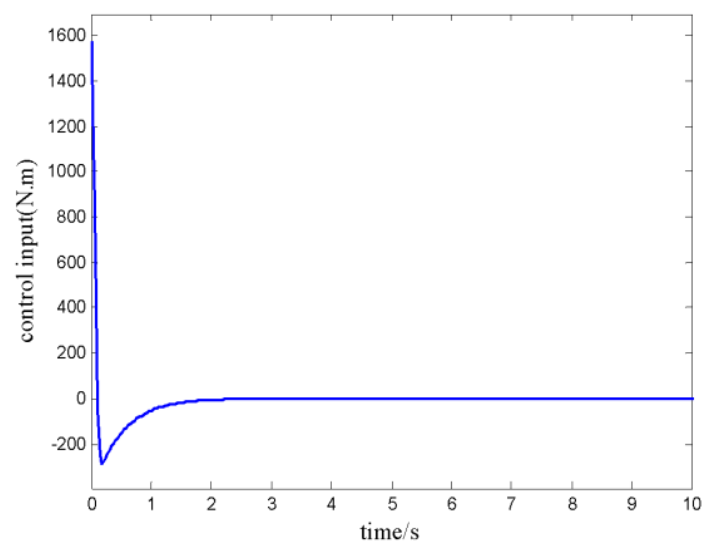

Figure. 5 control input of joint 1

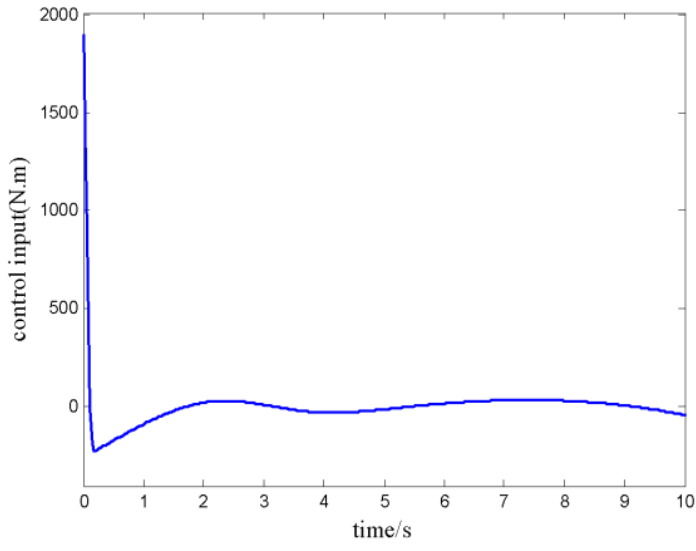

Figure. 6 control input of joint 2

\section{Summary}

An adaptive fuzzy sliding mode robust control algorithm based on fuzzy compensation is proposed for the uncertain items. It can be seen from the simulation results that the actual output of the system can track the reference trajectory with good location tracking and speed tracking performance. The uncertainty of the system and the influence of the external disturbance can be eliminated. The simulation results demonstrate the effectiveness of the proposed adaptive fuzzy robust controller for the uncertain nonlinear system.

\section{References}

[1] Cheng L, Hou Z G, Tan M, Adaptive neural network tracking control for manipulators with uncertain kinematics, dynamics and actuator model J. Automatica, 2009, 45(10):2312-2318.

[2] Wai R J, Muthusamy R, Fuzzy-neural-network inherited sliding-mode control for robot manipulator including actuator dynamics J.IEEE Transactions on Neural Networks and Learning Systems, 2013, 24(2): 274-287.

[3] Yao B, Tomizuka M, Adaptive robust control of SISO nonlinear systems in a semi-strict feedback form J. Automatica, 1997, 33(5):893-900.

[4] M.Zhihong and M.Palaniswami.A, Robust tracking control scheme for rigid roboticmanipulators with uncertain dynamics J. Computers \& Electrical Engineering, vol. 21,1995: 211-220Manufacturing, 2013, 3(2): 51-72.

[5] Kolhe J P, Shaheed M, Chandar T S, et al. Robust control of robot manipulators based on uncertainty and disturbance estimation J. International Journal of Robust and Nonlinear Control, 2013, 23(1): 104-122.

[6] Azadi M, Fazelzadeh S A, Eghtesad M, et al, Vibration suppression and adaptive-robust control of a smart flexible satellite with three axes maneuvering J. Acta Astronautica, 2011,69(5):307-322.

[7] Garelli F, Gracia L, Sala A, et al, Sliding mode speed auto-regulation technique for robotic tracking J. Robotics and Autonomous Systems, 2011,59(7):519-529

[8] Ertugrual M, Kaynak O. Neuro sliding mode control of robot manipulators J. Mechatronics,2000,10(1-2): 230-263. 